\author{
Katarzyna Kozak, Mateusz Spałek
}

Maria Sklodowska-Curie Institute - Oncology Centre, Warsaw, Poland

\title{
Perioperative management of soft tissue sarcomas
}

\author{
Address for correspondence: \\ Lek. Katarzyna Kozak \\ Lek. Mateusz Spałek \\ Centrum Onkologii — Instytut \\ im. Marii Skłodowskiej-Curie w Warszawie \\ e-mail:mateusz@spalek.co; \\ wiatrowka@gmail.com
}

Oncology in Clinical Practice

2018, Vol. 14, No. 6, 302-306

DOI: 10.5603/OCP.2018.0044

Translation: dr n. med. Dariusz Stencel

Copyright (๑ 2018 Via Medica

ISSN 2450-1654

\begin{abstract}
Surgery is the standard treatment of all patients with an adult type, localized soft tissue sarcoma. The wide excision is followed by radiation therapy as the standard treatment of high-grade (G2-3), deep, > $5 \mathrm{~cm}$ lesions. The goal of (neo)adjuvant radiation therapy is to improve local control, avoid amputation and preserve tissue by limiting the extent of resection. There is no consensus on the current role of adjuvant chemotherapy in localized soft tissue sarcoma. Given the conflicting results of trials and meta-analyses, neoadjuvant chemotherapy can be proposed as an option to the high-risk individual patient.
\end{abstract}

Key words: soft tissue sarcoma, adjuvant radiotherapy, adjuvant chemotherapy

Oncol Clin Pract 2018; 14, 6: 302-306

\section{Introduction}

Progress in the management of soft tissue sarcomas, which has taken place in primary treatment as well as in recurrent disease, is the result of multimodal procedures conducted in specialised centres. The combination of surgical treatment (the primary method) with radiotherapy and sometimes chemotherapy is a standard of care that should be planned and carried out by multidisciplinary teams. Modern combination therapy, including reconstructive procedures, allows the limb to be saved in the majority of patients with soft tissue sarcomas of the aforementioned location.

\section{Radiotherapy}

Perioperative radiotherapy is part of the combined limb-sparing treatment of soft tissue sarcomas. The results of randomised clinical trials have demonstrated the efficacy of such a procedure in obtaining satisfactory local control (approximately 90\%) and a similar survival time as in patients undergoing amputation $[1,2]$.

Perioperative radiotherapy (preoperative or postoperative) is indicated in case of subfascially located sarcomas stage IB-III (T2-T4 or G2-G3) [3]. Irradiation should also be considered in the following situations:

- non-radical resection of sarcomas stage IA (R1/R2, microscopic surgical margin less than $1 \mathrm{~mm}$, tumour damage during surgery);

- non-radical surgery of sarcomas located suprafascially (superficially) without the possibility of further re-resection;

- sarcomas of the head and neck region and trunk;

- the need to perform a re-operation (after a non-radical excision or after confirmed relapse).

The whole treatment (radiotherapy and surgery) should be carried out in one centre with equipment and a staff experienced in the treatment of these cancers. Radiation therapy of sarcomas requires individualisation in each patient. Due to the high heterogeneity of anatomical locations, tumour dimensions, and related ailments (bleeding, pain, oedema, limitation of mobility), immobilisation for radiotherapy should be performed by a team of experienced technicians under the supervision of a physician and medical physicists who will perform a radiation treatment plan. Good reproducibility can be obtained through the use of appropriate equipment (thermoplastic mask, vacuum mattresses, optical position control systems) and pharmacological support (an- 

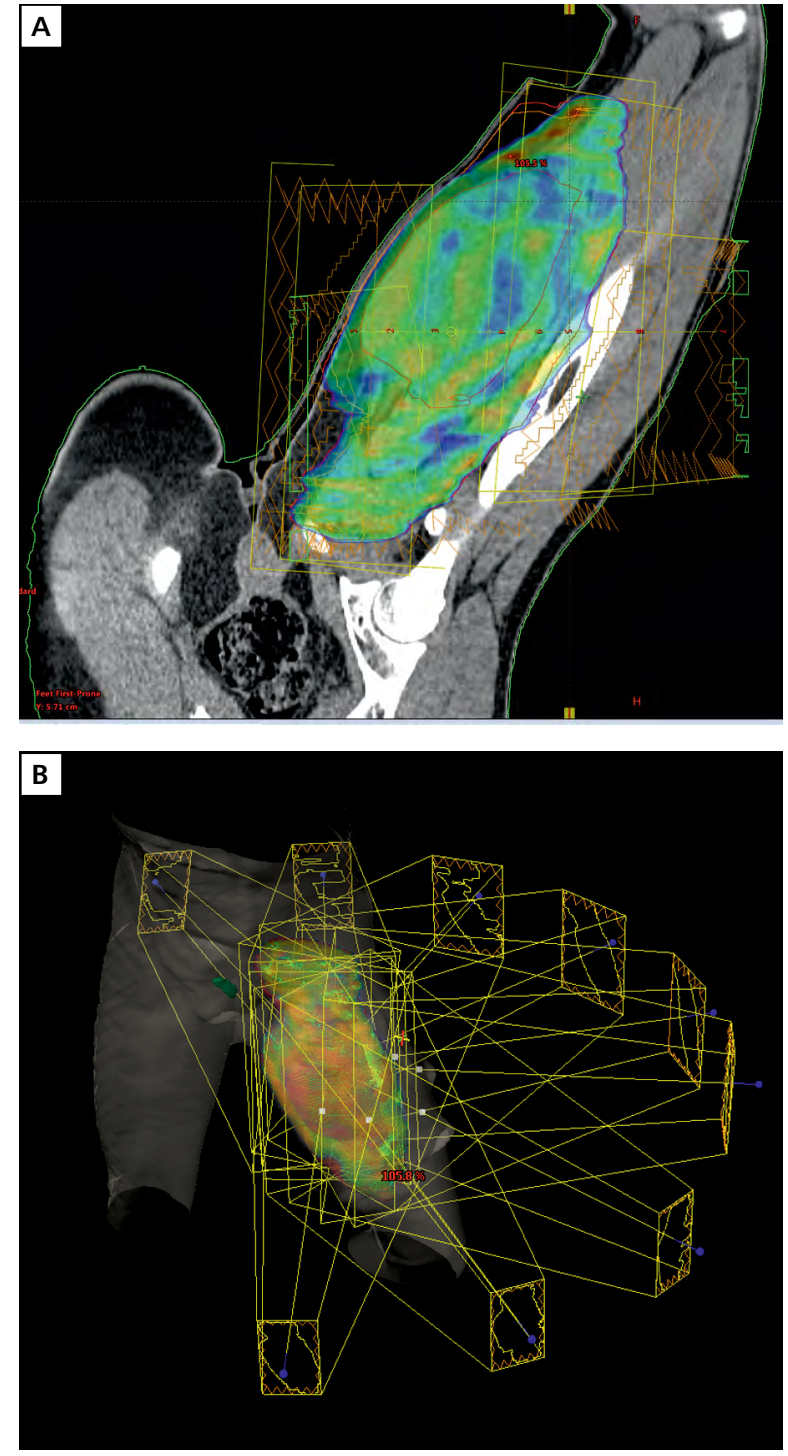

Figure 1. Plan of irradiation for locally advanced liposarcoma with the use of dynamic techniques. Source: own materials

algesics, anti-oedema, anxiolytics, myorelaxant agents). The choice of irradiation technique should take into account the location of the tumour, the proximity of critical organs, and the possibility of saving healthy tissues. The implementation of the treatment plan more and more often uses dynamic techniques (intensity-modulated radiation therapy [IMRT], volumetric modulation arc radiotherapy, Fig. 1), which may be beneficial in reducing the toxicity of treatment [4].

The choice of the sequence of radiotherapy and surgery remains unresolved. A clinical trial with random selection of patients did not show differences in local efficacy and survival between patients with preoperative and postoperative sarcoma [5]. Recruitment for the study was completed prematurely due to a higher rate of wound complications in patients receiving preoperative radiotherapy. Further observation of this cohort showed that although early complications from the postoperative wound occur more frequently in preoperatively irradiated patients, late complications of radiotherapy (fibrosis, joint stiffness, chronic oedema) developed in a higher percentage of patients irradiated postoperatively than preoperatively.

The benefits of preoperative radiotherapy include the possibility of more precise determination of target volume in the form of present tumour, removal of healthy tissues from the irradiated volume, lower risk of sarcoma cells implantation into the surgical wound, facilitation of sparing surgery, a smaller percentage of late complications, and a lower total dose in comparison to postoperative radiotherapy. Nevertheless, preoperative radiotherapy is associated with high risk of postoperative complications in the form of impaired wound healing (hence close cooperation between the radiotherapist and surgeon is required) and postpones the operation itself by several weeks, which increases the risk of disease progression.

In preoperative radiotherapy, gross tumour volume (GTV) is determined with the use of computed tomography (CT) for radiotherapy planning fused with magnetic resonance imaging with contrast agent. The elective clinical tumour volume (CTV) is determined by adding $1.5-2.0 \mathrm{~cm}$ around the GTV, taking into account the natural anatomical barriers (e.g. bones, fascia), while in case of extremity sarcomas the CTV is extended additionally in the longitudinal dimension up to $4 \mathrm{~cm}$ from the GTV poles. An error margin of $0.5-1.0 \mathrm{~cm}$ is added to CTV depending on the locally used protocol. The conventional fractionation scheme for preoperative radiotherapy is $50-50.4$ Gy in fractions of 1.8-2.0 Gy, followed by a 4-8-week break for surgery. In many centres, attempts are being made to apply higher fractional doses (up to $5 \mathrm{~Gy}$ ) and to combine irradiation with chemotherapy or targeted therapy. The advantages of hypofractionated preoperative radiotherapy include shortening the total time of treatment, greater comfort for the patient and doctor, and theoretically higher biological effectiveness due to the low values of the alpha/beta ratio of most sarcomas. A potential threat is the higher risk of late radiation-related complications (fibrosis, joint stiffness, bone fractures, neuropathies) compared to conventionally fractionated preoperative radiotherapy. Preliminary results indicate high efficacy and good tolerance of such procedures; however, attempts to change the fractionation and association of radiotherapy with systemic treatment should take place under the conditions of prospective clinical trials in reference centres for sarcoma treatment [6].

The advantages of postoperative radiotherapy include avoiding the complications of wound healing 
as well as not delaying surgery for the period of irradiation and breaks for the procedure. In the case of postoperative radiotherapy, a fusion of CT for planning radiotherapy with preoperative imaging examinations is required. It is possible to use modern techniques of flexible fusion in order to better reproduce the position of the tumour in postoperative anatomical conditions. The primary gross tumour volume (CTVg) is a tumour visible in preoperative studies together with the tumour bed after resection. Elective high-risk disc volume (CTVh) is determined by adding a $1.0-1.5-\mathrm{cm}$ margin of healthy tissue around the tumour. Elective medium-risk disc volume $(\mathrm{CTVm})$ is the same as in preoperative radiotherapy, adding $1.5-2.0 \mathrm{~cm}$ around CTVg including natural anatomical barriers (e.g. bones, fascia) and additionally in the longitudinal dimension up to $4 \mathrm{~cm}$ from the CTVm poles in the case of sarcomas of extremities. A margin of $0.5-1.0 \mathrm{~cm}$ is added to each of the elective disc volumes (CTVh, CTVm). The conventional fractionation scheme assumes the administration of $50 \mathrm{~Gy}$ in fractions of $2 \mathrm{~Gy}$ per indirect risk volume and, in the second stage of treatment, an additional dose (boost) only for the high-risk area. The dose in the second stage of treatment depends on the outcome of the operation: $10 \mathrm{~Gy}$ in 2-Gy fractions in the case of R0 surgery, and 16 Gy in 2-Gy fractions in the case of non-radical surgery or the presence of other relapse risk factors.

A special group comprises patients with locally advanced, border resectable, or unresectable soft tissue sarcomas with the presence of dissemination risk factors, but without metastases (high grade of histological malignancy, tumour size $>10 \mathrm{~cm}$, infiltration of structures that prevent radical resection, for example the cerebrovascular bundles). In such cases, patients should be offered participation in prospective clinical trials with preoperative radiotherapy combined with other treatment modalities (chemotherapy, targeted therapy, hyperthermia).

Typical early complications of irradiation include:

- complications in wound healing;

- swelling;

— skin reactions including necrosis (Fig. 2).

Late complications of irradiation include:

- fibrosis of the skin and subcutaneous tissue;

- deformations as well as poor functional and cosmetic treatment effect;

- limitation of joint mobility;

- chronic lymphatic oedema;

- bone fracture;

- nerve damage;

- complications associated with irradiation of critical organs located near the disc volume (e.g. rectum, lungs). Radiotherapy of small-round-cell sarcomas should be discussed separately; for detailed information see the report for Ewing sarcomas.

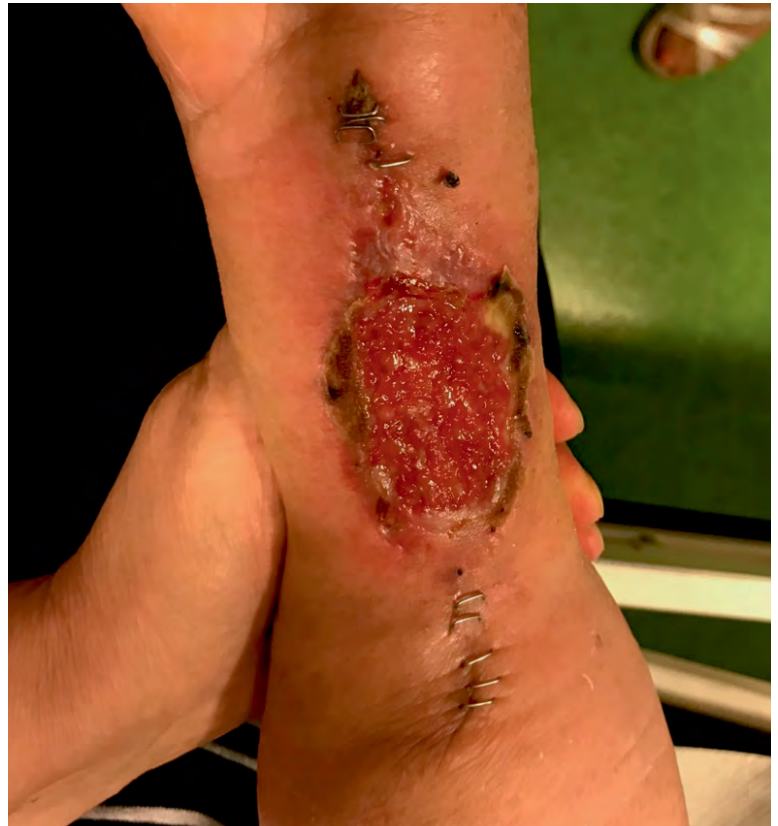

Figure 2. Complications in wound healing in a preoperatively irradiated patient. Source: own materials

\section{Systemic treatment}

The role of adjuvant chemotherapy in adult patients diagnosed with locally advanced soft tissue sarcomas (except for small-cell sarcoma and rhabdomyosarcoma) is not clearly defined. Due to the rare occurrence and heterogeneity of this group of tumours, there is a lack of well-designed, prospective studies with random selection of patients, the results of which could become the basis for establishing the current standard of care.

A meta-analysis (The Sarcoma Meta-Analysis Collaboration) published in 1997, including 14 randomised clinical trials and 1568 soft-tissue sarcoma patients undergoing doxorubicin-based postoperative chemotherapy, showed an improvement in 10-year rate of local $(81 \%$ vs. $75 \%, \mathrm{p}=0.016)$ and distant relapse-free survival $(70 \%$ vs. $60 \%, \mathrm{p}=0.0003)$. The use of adjuvant treatment improved 10-year relapse-free survival by $10 \%$ (significant difference) and overall survival by about $6 \%$ (non-significant difference). The effect on survival did not depend on the adjuvant therapy regimen used (monotherapy vs. multidrug regimens) [7]. A second meta-analysis published 11 years later included four randomised clinical trials, so the cumulative data derive from a total of 18 clinical trials, in which 1953 soft tissue sarcoma patients received adjuvant doxorubicin-based chemotherapy. The results of this meta-analysis confirmed the effect of adjuvant chemotherapy on relapse-free survival and both local and distant metastases. Moreover, a small but significant effect on the over- 
all survival of patients was demonstrated. An analysis including all studies showed a death risk reduction by $6 \%(95 \% \mathrm{CI} 2-11 \%, \mathrm{p}=0.003)$, and an analysis including only studies with adjuvant doxorubicin-ifosfamide combination showed a death risk reduction by $11 \%(95 \%$ CI 3-19\%; $\mathrm{p}=0.01$ ) [8].

These positive meta-analyses were not confirmed in the two large phase III randomised trials conducted by EORTC. In the EORTC 62931 study, patients $(n=351)$ after macroscopically radical surgical resections of soft tissue sarcomas at any location (histological malignancy grade $2-3$ ) received postoperatively five cycles of doxorubicin with ifosfamide or remained under observation. Interim analysis showed no effect of adjuvant chemotherapy, not only on overall survival, but also on relapse-free survival [9]. Similar results were obtained in the previous EORTC 62771 study, in which patients underwent eight cycles of post-operative chemotherapy according to CYVADIC (cyclophosphamide, vincristine, doxorubicin, dacarbazine) regimen or observation alone. The use of adjuvant chemotherapy improved only the relapse-free survival $(56 \% \mathrm{vs} .43 \%$, $\mathrm{p}=0.007)$ and decreased the rate of local recurrence (17\% vs. $31 \%, \mathrm{p}=0.004)$. There was no effect on distant relapse-free survival and overall survival [10]. In the meta-analysis of these two trials, both relapse-free survival and overall survival were better only in patients after $\mathrm{R} 1$ resection [11].

Due to lack of unambiguous data, the appropriate sequence of possible adjuvant treatment is not determined (pre-, peri-, or postoperative chemotherapy). In a clinical trial of the Italian and Spanish sarcoma group, the effectiveness of only preoperative chemotherapy (three cycles of epirubicin with ifosfamide) was compared with perioperative chemotherapy (three cycles before and two cycles after surgery, according to the same regimen) [12]. With a median follow-up of more than 10 years, the use of chemotherapy only before surgery was not associated with a worse prognosis [13]. Better overall survival was observed in patients who responded to chemotherapy (in particular assessed according to Choi criteria) [14].

The advantage of preoperative chemotherapy, especially when used in combination with radiotherapy, is the improvement of the effectiveness of surgical treatment. This is particularly important in case of large tumours with borderline resection and sparing surgery. The disadvantage, however, may be the delay of surgical treatment in the case of resistance to chemotherapy. Therefore, during the preoperative treatment, the patient should be carefully observed, and if the tumour progresses, radical local treatment should be carried out immediately.

An additional option for soft tissue sarcoma patients with extremities localisation and high local advance- ment may be the use of isolated limb perfusion (ILP) with anti-cancer drugs (HILP, hyperthermic isolated limb perfusion). This procedure may facilitate surgical treatment but does not affect the overall survival of patients [15].

In addition to the different treatment sequences, there are also different regimens of adjuvant chemotherapy, mainly based on anthracyclines [16]. The Phase III study carried out with the cooperation of four sarcoma groups (French, Spanish, Polish, and Italian) aimed to assess the effectiveness of neoadjuvant chemotherapy adjusted to the histological subtype of the sarcoma. The study included 287 patients with soft tissue sarcoma of limbs or trunk. The patients were assigned to the group receiving three cycles of either epirubicin with ifosfamide or chemotherapy dedicated to a given histological subtype: liposarcoma - trabectedin, leiomyosarcoma - gemcitabine with dacarbazine, synovial sarcoma - ifosfamide, MPNST - etoposide with ifosfamide, pleomorphic sarcoma - gemcitabine with docetaxel. The study was prematurely closed because three subsequent interim analyses showed significantly better relapse-free survival as well as overall survival in patients receiving epirubicin plus ifosfamide [17].

Given the conflicting results of clinical trials and meta-analyses, adjuvant chemotherapy should only be considered in some patients with locally advanced soft tissue sarcomas (tumour diameter over $5 \mathrm{~cm}$, high histological grade, subfascial location) together with possible toxicity of treatment. Radiotherapy should not delay the onset of chemotherapy, and it can be successfully used in combination with pre-operative chemotherapy [18-20].

\section{References}

1. Rosenberg SA, Tepper J, Glatstein E, et al. The treatment of soft-tissue sarcomas of the extremities: prospective randomized evaluations of (1) limb-sparing surgery plus radiation therapy compared with amputation and (2) the role of adjuvant chemotherapy. Ann Surg. 1982; 196(3): 305-315, indexed in Pubmed: 7114936

2. Yang JC, Chang AE, Baker AR, et al. Randomized prospective study of the benefit of adjuvant radiation therapy in the treatment of soft tissue sarcomas of the extremity. J Clin Oncol. 1998; 16(1): 197-203, doi: 10.1200/JCO.1998.16.1.197, indexed in Pubmed: 9440743.

3. Rutkowski P, Ługowska I, Fijuth J, et al. Soft tissue sarcomas in adults. Oncol Clin Pract 2017; 13: 181-201. DOI: 10 5603/OCP. ; 2017: 0025, doi: 10.5603/OCP.2017.0025

4. O'Sullivan B, Griffin AM, Dickie Cl, et al. Phase 2 study of preoperative image-guided intensity-modulated radiation therapy to reduce wound and combined modality morbidities in lower extremity soft tissue sarcoma. Cancer. 2013; 119(10): 1878-1884, doi: 10.1002/cncr.27951, indexed in Pubmed: 23423841

5. O'Sullivan B, Davis AM, Turcotte R, et al. Preoperative versus postoperative radiotherapy in soft-tissue sarcoma of the limbs: a randomised trial. Lancet. 2002; 359(9325): 2235-2241, doi 10.1016/S01406736(02)09292-9, indexed in Pubmed: 12103287.

6. Haas RLM, Miah AB, LePechoux C, et al. Preoperative radiotherapy for extremity soft tissue sarcoma; past, present and future perspectives on dose fractionation regimens and combined modality strategies. Radiother Oncol. 2016; 119(1): 14-21, doi: 10.1016/j.radonc.2015.12.002, indexed in Pubmed: 26718153. 
7. Adjuvant chemotherapy for localised resectable soft-tissue sarcoma of adults: meta-analysis of individual data. Sarcoma Meta-analysis Collaboration. Lancet. 1997; 350(9092): 1647-1654, indexed in Pubmed: 9400508

8. Pervaiz N, Colterjohn N, Farrokhyar F, et al. A systematic meta-analysis of randomized controlled trials of adjuvant chemotherapy for localized resectable soft-tissue sarcoma. Cancer. 2008; 113(3): 573-581, doi 10.1002/cncr.23592, indexed in Pubmed: 18521899.

9. Woll PJ, Reichardt P, Le Cesne A, et al. EORTC Soft Tissue and Bone Sarcoma Group and the NCIC Clinical Trials Group Sarcoma Disease Site Committee. Adjuvant chemotherapy with doxorubicin, ifosfamide, and lenograstim for resected soft-tissue sarcoma (EORTC 62931): a multicentre randomised controlled trial. Lancet Oncol. 2012; 13(10): 1045-1054 doi: 10.1016/S1470-2045(12)70346-7, indexed in Pubmed: 22954508

10. Bramwell V, Rouesse J, Steward W, et al. Adjuvant CYVADIC chemotherapy for adult soft tissue sarcoma-reduced local recurrence bu no improvement in survival: a study of the European Organization for Research and Treatment of Cancer Soft Tissue and Bone Sarcoma Group. J Clin Oncol. 1994; 12(6): 1137-1149, doi: 10.1200/ JCO.1994.12.6.1137, indexed in Pubmed: 8201375.

11. Le Cesne A, Ouali M, Leahy MG, et al. Doxorubicin-based adjuvan chemotherapy in soft tissue sarcoma: pooled analysis of two STBSG-EORTC phase III clinical trials. Ann Oncol. 2014; 25(12): 2425--2432 doi: 10.1093/annonc/mdu460, indexed in Pubmed: 25294887.

12. Gronchi A, Frustaci S, Mercuri M, et al. Short, full-dose adjuvant chemotherapy in high-risk adult soft tissue sarcomas: a randomized clinical trial from the Italian Sarcoma Group and the Spanish Sarcoma Group. J Clin Oncol. 2012; 30(8): 850-856, doi: 10.1200/JC0.2011.37.7218, indexed in Pubmed: 22312103.

13. Gronchi A, Stacchiotti S, Verderio P, et al. Short, full-dose adjuvant chemotherapy (CT) in high-risk adult soft tissue sarcomas (STS): long-term follow-up of a randomized clinical trial from the Italian Sarcoma Group and the Spanish Sarcoma Group. Ann Oncol. 2016; 27(12): 2283-2288 doi: 10.1093/annonc/mdw430, indexed in Pubmed: 27733375
14. Stacchiotti S, Verderio P, Messina A, et al. Tumor response assessment by modified Choi criteria in localized high-risk soft tissue sarcoma treated with chemotherapy. Cancer. 2012; 118(23): 5857-5866, doi: 10.1002/cncr.27624, indexed in Pubmed: 22605504

15. Deroose JP, Eggermont AMM, van Geel AN, et al. Long-term results of tumor necrosis factor alpha- and melphalan-based isolated limb perfusion in locally advanced extremity soft tissue sarcomas. J Clin Oncol. 2011: 29(30): 4036-4044, doi: 10.1200/JCO.2011.35.6618, indexed in Pubmed: 21931039.

16. Rothermundt C, Fischer GF, Bauer S, et al. Pre- and Postoperative Chemotherapy in Localized Extremity Soft Tissue Sarcoma: A European Organization for Research and Treatment of Cancer Expert Survey. Oncologist. 2018; 23(4): 461-467, doi: 10.1634/theoncologist.2017-0391, indexed in Pubmed: 29192019.

17. Gronchi A, Ferrari S, Quagliuolo V, et al. Histotype-tailored neoadjuvant chemotherapy versus standard chemotherapy in patients with high-risk soft-tissue sarcomas (ISG-STS 1001): an international, open-label, randomised, controlled, phase 3, multicentre trial. Lancet Oncol. 2017; 18(6): 812-822, doi: 10.1016/S1470-2045(17)30334-0, indexed in Pubmed: 28499583

18. Palassini E, Ferrari $S$, Verderio $P$, et al. Feasibility of Preoperative Chemotherapy With or Without Radiation Therapy in Localized Soft Tissue Sarcomas of Limbs and Superficial Trunk in the Italian Sarcoma Group/Grupo Español de Investigación en Sarcomas Randomized Clinical Trial: Three Versus Five Cycles of Full-Dose Epirubicin Plus Ifosfamide. J Clin Oncol. 2015; 33(31): 3628-3634, doi: 10.1200/ JCO.2015.62.9394, indexed in Pubmed: 26351345.

19. Casali PG, Abecassis N, Bauer S, et al. Soft tissue and visceral sarcomas: ESMO-EURACAN Clinical Practice Guidelines for diagnosis, treatment and follow-up. Ann Oncol 2018; 29 (Suppl 4): iv51-iv67. doi: 10.1093/annonc/mdy096.

20. Rutkowski P, Ługowskal, Fijuth J, et al. Soft tissue sarcomas in adults. Oncol Clin Pract 2017: 13: 181-201, doi: 10.5603/OCP.2017.0025. 